\title{
Lymph node fibroblastic reticular cells deposit fibrosis- associated collagen following organ transplantation
}

\author{
Xiaofei Li, ${ }^{1,2}$ Jing Zhao, ${ }^{1}$ Vivek Kasinath, ${ }^{1}$ Mayuko Uehara, ${ }^{1}$ Liwei Jiang, ${ }^{1}$ Naima Banouni, ${ }^{1}$ Martina M. McGrath, ${ }^{2}$ \\ Takaharu Ichimura, ${ }^{3}$ Paolo Fiorina, ${ }^{1}$ Dario R. Lemos, ${ }^{3,4}$ Su Ryon Shin, ${ }^{5}$ Carl F. Ware, ${ }^{6}$ Jonathan S. Bromberg, ${ }^{7}$ and Reza Abdi ${ }^{1}$ \\ ${ }^{1}$ Transplantation Research Center, Renal Division, Brigham and Women's Hospital, Harvard Medical School, Boston, Massachusetts, USA. ${ }^{2}$ Key Laboratory of Combinatorial Biosynthesis and Drug Discovery, \\ Wuhan University School of Pharmaceutical Sciences, Wuhan, Hubei, China. ${ }^{3}$ Renal Division, Brigham and Women's Hospital, ${ }^{4}$ Harvard Stem Cell Institute, and ${ }^{5}$ Division of Engineering in Medicine, Brigham \\ and Women's Hospital, Harvard Medical School, Boston, Massachusetts, USA. ${ }^{6}$ Infectious and Inflammatory Diseases Center, Sanford Burnham Prebys Medical Discovery Institute, La Jolla, California, USA. \\ 7Department of Surgery and Microbiology and Immunobiology, University of Maryland School of Medicine, Baltimore, Maryland, USA.
}

\begin{abstract}
Although the immune response within draining lymph nodes (DLNs) has been studied for decades, how their stromal compartment contributes to this process remains to be fully explored. Here, we show that donor mast cells were prominent activators of collagen I deposition by fibroblastic reticular cells (FRCs) in DLNs shortly following transplantation. Serial analysis of the DLN indicated that the LN stroma did not return to its baseline microarchitecture following organ rejection and that the DLN contained significant fibrosis following repetitive organ transplants. Using several FRC conditional-knockout mice, we show that induction of senescence in the FRCs of the DLN resulted in massive production of collagen I and a proinflammatory milieu within the DLN. Stimulation of herpes virus entry mediator (HVEM) on FRCs by its ligand LICHT contributed chiefly to the induction of senescence in FRCs and overproduction of collagen I. Systemic administration of ex vivo-expanded FRCs to mice decreased DLN fibrosis and strengthened the effect of anti-CD40L in prolonging heart allograft survival. These data demonstrate that the transformation of FRCs into proinflammatory myofibroblasts is critically important for the maintenance of a proinflammatory milieu within a fibrotic DLN.
\end{abstract}

\section{Introduction}

Lymph nodes (LNs) are the quintessential organs of alloimmunity following transplantation. Recent advances in elucidating the function of specific cellular and stromal compartments in the LN have broadened our understanding of how it controls the alloimmune response $(1,2)$. The stromal microenvironment within the $\mathrm{LN}$ is the critical arbiter of the balance between propagation and regulation of the immune response (3).

The stromal compartment of the LN is maintained by fibroblastic reticular cells (FRCs). FRCs are cells of mesodermal origin that support the structure of the $\mathrm{LN}$, and they control many facets of adaptive immunity $(4,5)$. FRCs classically express podoplanin (PDPN), but they do not express CD31 or CD45, a unique cellular signature in the LN. The homing of $\mathrm{T}$ cells to the $\mathrm{LN}$ and presentation of antigens to these T cells by dendritic cells (DCs) are prerequisites for the development of allorecognition $(6,7)$. These phenomena are supported fully and regulated by FRCs, which mold the microarchitecture of the LN and create its characteristic compartmentalization. FRCs produce chemokines that attract

\section{Related Commentary: p. 3965}

Authorship note: XL and JZ contributed equally to this work Conflict of interest: The authors have declared that no conflict of interest exists. Copyright: (C) 2020, American Society for Clinical Investigation. Submitted: January 21, 2020; Accepted: April 22, 2020; Published: June 29, 2020. Reference information: / Clin Invest. 2020;130(8):4182-4194. https://doi.org/10.1172/JCl136618.
$\mathrm{T}$ cells to the LN via migration through high endothelial venules (HEVs), and they control the integrity and permeability of these HEVs within the LN (8). Following their entry, T cells crawl on the scaffold built by FRCs either to locate antigen-presenting cells (APCs) or exit the LN (9). A key manifestation of the activation of LNs is increased synthesis of extracellular matrix (ECM), which is required to support an expansion of proliferating immune cells $(5,10,11)$. However, the immunosuppressive qualities of FRCs are especially important toward the late phase of an immune response, when the LN must involute to its steady-state functioning and microarchitecture to maintain immunological homeostasis and avoid sustained deleterious inflammation (12).

FRCs are derived from lymphoid tissue organizer cells in a lymphotoxin $\beta$ receptor-dependent (LT $\beta$ R-dependent) manner (13). In fact, mice that are completely deficient for the LT $\beta R$ lack LNs. In addition to LT $\alpha$, another ligand called lymphotoxin-like inducible protein that competes with glycoprotein D for herpes virus entry on T cells (LIGHT, also known as TNF superfamily member 14 [TNFSF14]) binds not only to the LT $\beta$ R but also to its counterpart, herpes virus entry mediator (HVEM, also known as TNF receptor superfamily member 14 [TNFRSF14]). HVEM and LIGHT are widely recognized for their functions as checkpoint regulators of T cells, but their roles in controlling the fate of FRCs remain largely unknown (14). Here, we tested the overall hypothesis that sustained activation of FRCs through HVEM on their cell membranes will result in their transformation into proinflammatory myofibroblasts, creating a proinflammatory milieu within the fibrotic LN that would promote alloimmunity. 
LN fibrosis has been reported previously in infectious models in which the pathogens have tropism for FRCs $(15,16)$. Previous studies by our group also demonstrated the importance of fibrosis in the kidney-draining LN (KLN) to the progression of renal disease following repetitive ischemia/reperfusion injury (IRI) to the kidney (17). Despite an enormous body of literature devoted to the characterization of various effector immune responses within the draining LN (DLN), almost no attention has been paid to the microstructural changes that occur within the DLN following transplantation or to determining whether its microarchitecture recovers to its resting state. Furthermore, no data are available on the presence of LN fibrosis in transplant tolerance, or its implication for this process. In this series of experiments, we demonstrated that ECM is produced in the stromal compartment of the DLN shortly following transplantation and that repetitive proinflammatory stimuli following successive transplantations activated the LIGHT/HVEM pathway, which induces the senescence-associated secretory phenotype (SASP) of FRCs, characterized by robust production of ECM as well as fibrosis in the DLN. Remarkably, administration of FRCs reduced senescence in FRCs and fibrosis in the DLN and also prolonged survival in murine cardiac allograft recipients.

\section{Results}

Mast cells as key regulators of early collagen I production in the DLN following skin transplantation. Skin allografts from BALB/c mice were transplanted onto C57BL/6 mice (WT, WT), and DLNs were harvested and sectioned to study the ECM as early as 1 day after transplantation. The DLNs contained a higher density of collagen I fibers as well as expanded lymphatic vessels in comparison with naive LNs $(P<0.01$, Figure $1 \mathrm{~A})$. We used a stepwise approach to investigate the role of various immune cells in the stimulation of FRCs to produce collagen I shortly after transplantation. We observed a similar increase in collagen I in the DLNs of $\mathrm{Rag1}^{-1-}$ recipients (Supplemental Figure 1A; supplemental material available online with this article; https://doi.org/10.1172/ JCI136618DS1), suggesting that the activities of alloreactive $\mathrm{T}$ and B cells were not critical for the induction of collagen I. Depletion of macrophages through the treatment of the transplant recipient with clodronate likewise did not affect collagen I synthesis in the DLNs (Supplemental Figure 1B). In addition, we observed no effect on Lyve-1+ lymphatic vessels or Meca-79+ ${ }^{+}$HEVs (data not shown). Mast cells are a family of early innate immune cells that could potentially induce the formation of collagen I in the DLN, so we investigated their possible role. Staining with toluidine blue revealed a significant increase in the density of mast cells in the DLNs of skin transplant recipients compared with LNs from naive mice $(P<0.05$, Figure 1B). Furthermore, the fluorescent signal of the type I high-affinity IgE receptor (FceR1), a protein specifically expressed by mast cells (18), was also higher in the DLNs compared with signal in naive LNs $(P<0.01$, Figure $1 C)$.

$\mathrm{Kit}^{\mathrm{w}-\mathrm{sh} / \mathrm{w}-\mathrm{sh}}$ mice (on a C57BL6 background), which lack mast cells, were used as recipients and donors to study the role of host and intragraft mast cells in the production of ECM after transplantation. Although the use of mast cell-deficient recipients resulted in no significant impact on collagen I deposition (data not shown), we observed a decrease in collagen I deposition in the DLNs when we used mast cell-deficient donors (as compared with WT donors $)(P<0.05$, Figure 1D and Supplemental Figure $1 C)$. To confirm the migration of mast cells, we transplanted skin allografts from C57BL/6-Tg (UBC-mCherry) mouse donors into $\mathrm{BALB} / \mathrm{c}$ recipients to determine whether mCherry ${ }^{+}$ cells infiltrated the DLNs of the recipient mice. We detected donor-derived DLN-infiltrating $\mathrm{mCherry}^{+}$cells that expressed FceR1 as well as tryptase (both mast cell markers) 2 hours after transplantation (Supplemental Figure 1D). As mast cells could respond quickly to the changes induced by IRI (19), we sought to determine whether donor mast cells alter the microarchitecture of the DLN in highly ischemic grafts. Remarkably, prolonged cold ischemia resulted in significant expansion of lymphatic vessels in the DLNs compared with the control DLNs $(P<0.05$, Supplemental Figure 1E). The ischemic DLNs also contained greater deposition of collagen I, collagen III, and collagen IV as compared with the control group (Supplemental Figure 1, E-G, $P<0.05$ for the collagen I and collagen III comparison; $P=0.05$ for the collagen IV comparison).

Next, we sought to determine the effect of ischemia on the secretory repertoire of P815 mast cells in vitro. Using in vitro oxidative stress (OS) assays $(20,21)$, the gene expression of murine mast cell proteases $M c p t 2, M c p t 4$, and $M c p t 6$, markers of mast cell activation (22), as well as of Vegfa, Fgf2, and Il6, was significantly higher in OS mast cells compared with expression levels in the control groups $(P<0.05$, Figure $1 \mathrm{E})$. Vegfa could support lymphatic expansion, Fgf2 could stimulate FRCs, and Il6 could create a proinflammatory milieu in the DLN, respectively. Culturing FRCs in media collected from P815 cells treated with $\mathrm{H}_{2} \mathrm{O}_{2}$ resulted in higher expression of collagen I (Col1a1), Tgfb1, and Smad2 by FRCs in comparison with expression in FRCs cultured in media extracted from untreated P815 cells $(P<0.05$, Figure $1 \mathrm{~F})$. We also examined the effect of mast cell-conditioned media on lymphangiogenesis in vitro using the SVEC4-10 cell line (23) and found that media from $\mathrm{H}_{2} \mathrm{O}_{2}$-treated mast cells resulted in more extensive tube formation in SVEC4-10 cells compared with media from untreated mast cells or no treatment (Figure 1G).

Accumulation of collagen I in the DLN long after rejection of the organ transplant. Next, we examined the deposition of collagen I fibers in the DLN following allograft rejection. Compared with a naive LN, the DLNs contained a higher density of collagen I fibers 7 days after skin transplantation. Interestingly, this accumulation persisted up to 42 days and 100 days after skin transplantation $(P<0.05$, Supplemental Figure $2 A)$. A similar increase in collagen I was also noted in the DLNs of heart transplant recipients $(P<0.05$, Supplemental Figure 2A). This finding suggests an absence of full recovery in the DLN microarchitecture following the complete rejection of the organ.

As many organ transplant recipients undergo second and third transplantations, we questioned whether retransplanting skin and heart allografts in the same location as the previous graft (following its full rejection) would result in amplification of collagen I deposition in the DLN within a short time frame. DLNs were harvested 4 weeks after the second heart transplant and third skin transplant in each mouse (Supplemental Figure 2B, referred to herein as $\mathrm{DLN}^{\mathrm{Rep}}$ ). We found that collagen I deposition in these DLNs became more dense following repetitive skin transplants as compared with a single transplant $(P<0.01$, Figure $2 \mathrm{~A}$ com- 



Figure 1. Mast cells as early inducers of collagen I deposition in the DLN. (A) Images show a comparison of collagen I (red) expression between the DLNs of mice on day 1 after allogeneic skin transplantation and the LNs of naive mice. Scale bars: $50 \mu \mathrm{m}$. Semiquantitative assessment is shown in the graph. $n=4$. (B) Toluidine blue staining of mast cells (dashed circles) and comparison of their populations in DLNs and naive LNs. Scale bars: $50 \mu \mathrm{m}$. $n=4$. (C) Representative IF staining of mast cells and semiquantitative assessment of Fc\&RI (green) expression by mast cells in DLNs. Lyve-1+ lymphatic endothelium (red) and DAPI+ nuclei (blue) staining is also shown. Scale bars: $50 \mu \mathrm{m} . n=4$. (D) IF staining of collagen I (red) and Lyve-1 (green) in the LNs of naive $\mathrm{BALB} / \mathrm{c}$ mice, as well as the DLNs of C57BL/ $6 \rightarrow \mathrm{BALB} / \mathrm{c}$ skin-transplanted mice and Kit ${ }^{\mathrm{W}-\mathrm{sh} / \mathrm{W}-\mathrm{sh}} \rightarrow \mathrm{BALB} / \mathrm{c}$ skin-transplanted mice. Scale bars: $50 \mu \mathrm{m}$. $n$ $=4$. (E) Gene expression levels of the mast cell proteases as well as Vegfa, Fgf2, and II6 with and without $\mathrm{H}_{2} \mathrm{O}_{2}$ stimulation ( $n=4$; each dot represents 1 sample). (F) Gene expression levels of Col1a1, Tgfb1, and Smad2 in FRCs following treatment with different mast cell-conditioned media (CM). $n=4$. (G) Micrographs and tube formation analysis of SVEC4-10 cells treated with different mast cell-conditioned media. Scale bars: $100 \mu \mathrm{m}$. The percentage of the areas stained positive in the fluorescence micrographs was assessed in 3-6 random microscopic fields for each mouse. Data are presented as the mean \pm SD. ${ }^{*} P<0.05$ and ${ }^{*} P<0.01$, by 2-tailed Student's $t$ test $(\mathbf{A}-\mathbf{C}$, and $\mathbf{E})$ and 2 -way ANOVA with Tukey's multiple comparisons post hoc test (F and $\left.\mathbf{G}\right)$.

pared with Supplemental Figure 2A). The DLN ${ }^{\text {Rep }}$ also contained thickened collagen I and fibronectin fibers in a nodular pattern, especially around the lymphatic vessels and HEVs, in contrast to the thin, fibrillary pattern observed in an age-matched naive LN (Figure 2B). Masson's trichrome staining also revealed a greater amount of fibrosis around the HEVs of the DLN ${ }^{\text {Rep }}$ compared with the naive LN (Figure 2C). We noted similar increases in collagen I deposition and lymphatic expansion in the DLN ${ }^{\text {Rep }}$ of heart transplant recipients ( 2 consecutive heart implantations in the abdomen, $P<0.05$, Supplemental Figure 2C). Electron micrographs of the DLN ${ }^{\text {Rep }}$ revealed the presence of mast cells, occlusion of HEVs with swelling of endothelial cells, and accumulation of collagen I fibers in the LN interstitium (Supplemental Figure 2D). Collagen I also accumulated in the spleens of mice that underwent repetitive skin transplantation (Supplemental Figure 2E). To understand the extent to which rejection contributed to collagen I deposition, syngeneic transplantation was performed in C57BL/6 mice as a comparator to the allogenic transplantation. The density of collagen I was slightly higher in the DLNs of the recipients of the syngeneic transplant as compared with the corresponding LNs of a naive mouse. However, collagen I deposition in the DLNs of the syngeneic transplant recipients was considerably lower than what we observed in the DLNs of the allogeneic transplant recipients (Supplemental Figure 2F). 
A
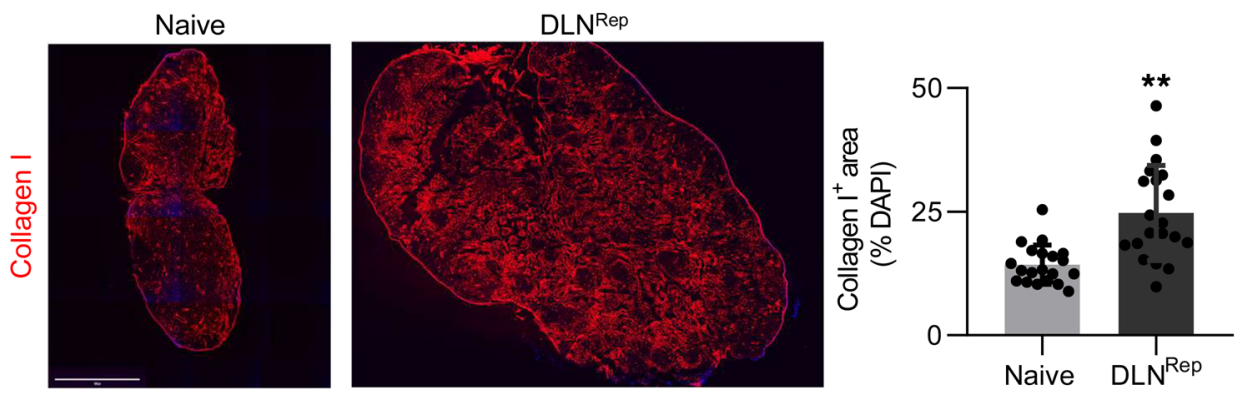

B
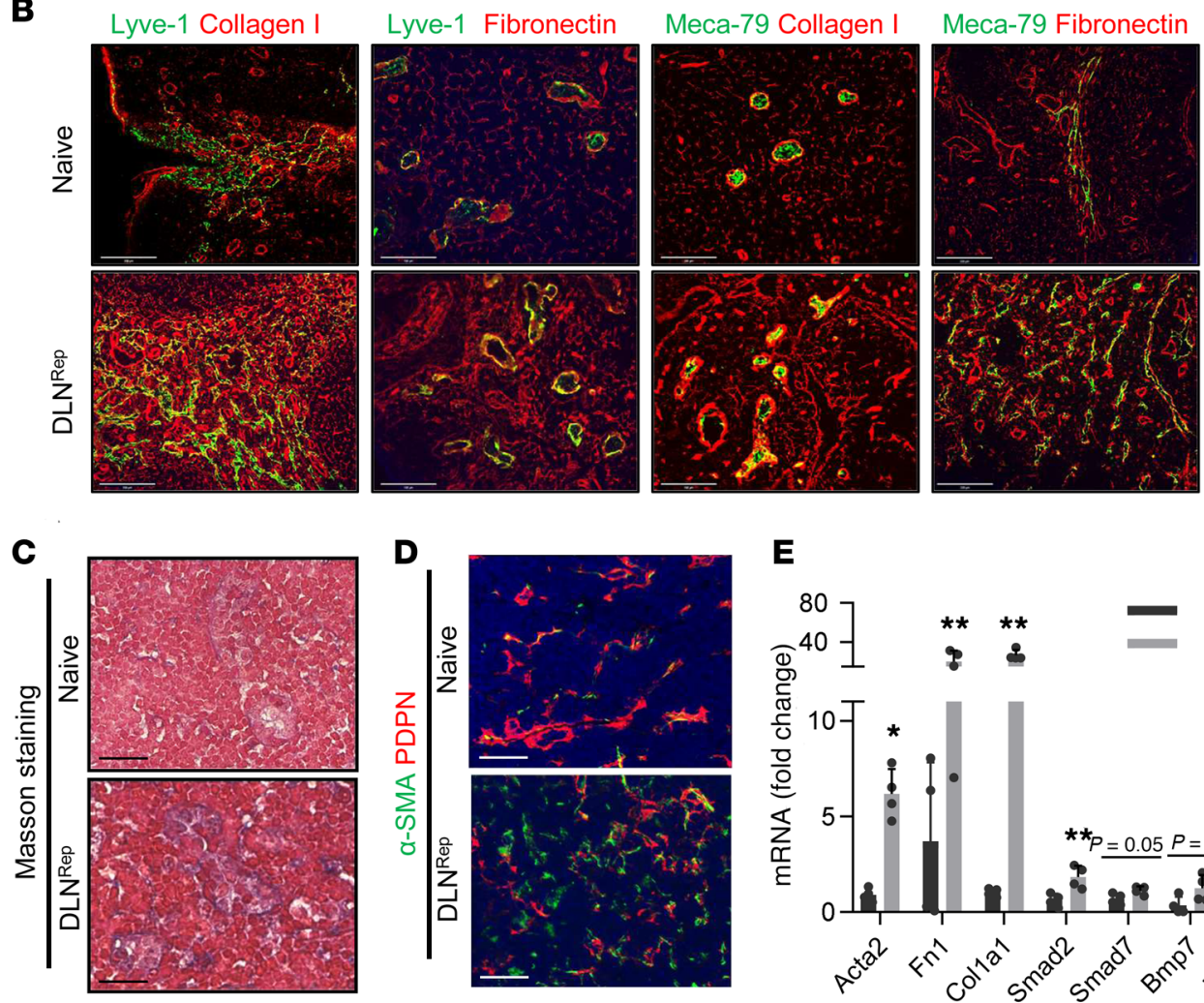

Figure 2. Repetitive skin transplantation induces fibrosis in DLNs. (A) Collagen I fibers (red) in the DLNs (axillary LNs, DLN ${ }^{\text {Rep})}$ of mice following repetitive skin transplantation in comparison with the axillary LNs of agematched naive mice and semiquantitative analysis. Scale bar: $1500 \mu \mathrm{m} . n=5$. (B) Costaining of Meca-79+ HEVs (green) and Lyve-1+ lymphatic vessels (green) with collagen I fibers (red) and fibronectin fibers (red) in DLN Rep and age-matched naive LNs. Scale bars: $100 \mu \mathrm{m}$. (C) Masson's trichrome stain of fibrosis in DLN $^{R e p}$ and age-matched naive LNs. Scale bars: $50 \mu \mathrm{m}$. (D) Fluorescence micrographs showing expression of the myofibroblast marker $\alpha$-SMA by PDPN ${ }^{+}$FRCs in $\mathrm{DLN}^{\mathrm{Rep}}$ and naive LNs. Scale bars: $100 \mu \mathrm{m}$. (E) Gene expression levels of fibrosis markers and TCF- $\beta$ signaling molecules in the DLNRep and $\mathrm{LNs}$ of naive mice. $n=4$. The percentage of the areas stained positive in the fluorescence micrographs was assessed in 3-6 random microscopic fields for each mouse. Data are presented as the mean $\pm \mathrm{SD}$. ${ }^{*} P<0.05$ and ${ }^{* *} P<0.01$, by Student's $t$ test.
In addition, $\alpha$-SMA, a marker of differentiated myofibroblasts (24), was detected by immunofluorescence (IF) staining. The number of $\mathrm{PDPN}^{+} \alpha-\mathrm{SMA}^{+}$cells was significantly higher in $\mathrm{DLN}^{\mathrm{Rep}}$ compared with naive LNs, indicative of the transition of FRCs toward myofibroblasts (Figure 2D and Supplemental Figure 2G). mRNA expression levels of ECM markers such as Acta2, Fn1, and Col1a1, the proinflammatory cytokine Tgfb1, and the key TGF- $\beta 1$ signaling molecule Smad2 were also increased in the DLN ${ }^{\text {Rep }}$ as compared with naive LNs (Figure 2E). We made similar observations in the para-aortic and mediastinal LNs, which functioned as the DLN ${ }^{\text {Rep }}$ following 2 consecutive heart transplants in the abdomen (data not shown).

Senescence in FRCs is associated with deposition of collagen I in the $D L N^{R e p}$. The process of senescence induces the proinflammatory secretome of cells, and unabated stress to these cells can result in the prolonged presence of senescent cells in a tissue, which can contribute significantly to tissue fibrosis and damage via multiple routes as a result of the persistence of their associated proinflammatory features (25). We have demonstrated previously that FRCs in the KLN become senescent following repetitive ischemia/reperfusion of the kidney (17). Next, we decided to test whether FRCs stimulated by strong and persistent proinflammatory cues become senescent and whether this transition affects their differentiation toward scar-forming myofibroblasts responsible for the development of fibrosis in the DLN following transplantation. The senescence marker $\beta$-gal was expressed more robustly in $\mathrm{DLN}^{\mathrm{Rep}}$ in comparison with naive LNs $(P=0.08$, Figure 3A and Supplemental Figure 3A). Sections of these LNs were also costained for the senescence marker p21 and the FRC marker PDPN, revealing that FRCs in the DLN ${ }^{\text {Rep }}$ expressed a significantly higher level of p21 than did those in a naive $\mathrm{LN}(P<$ 0.01 , Figure 3B and Supplemental Figure 3B). Additionally, the DLN $^{\text {Rep }}$ expressed higher mRNA levels of the senescence gene markers Cdkn2a, Cdkn1a, Trp53, and Cdkn1c ( $P<0.05$, Figure 3C).

Then, we isolated FRCs from the DLN ${ }^{\text {Rep }}$ and found that they expressed $\beta$-gal at higher levels than did FRCs isolated from the LNs of naive mice $(P<0.01$, Figure 3D and Supplemental Figure 3C). We also found that these FRCs expressed the senescence pro- 
A

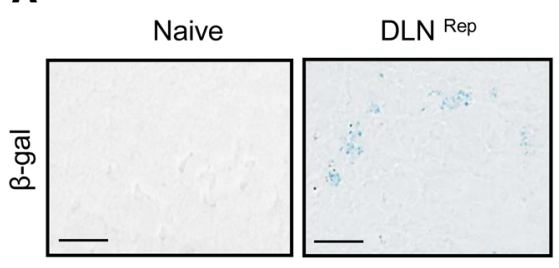

B
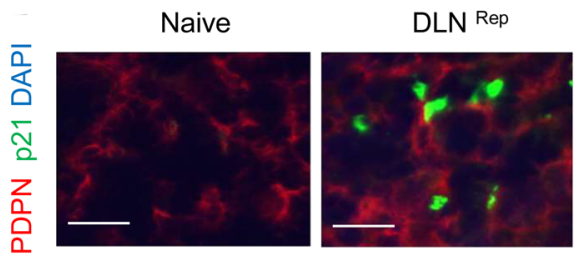

D

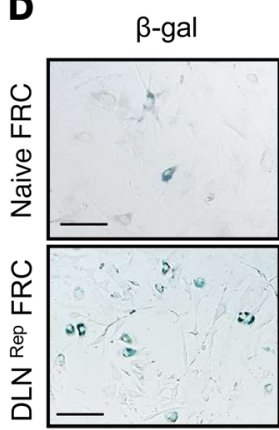

G

IDTR CCL19 cre

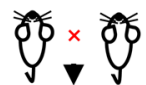<smiles>[C]1[C+]=C1</smiles>

CCL19 19 re $\times$ iDTR

J

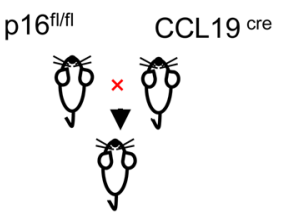

CCL19cre $\times$ p $16^{\text {fflf }}$
$\mathbf{E}$
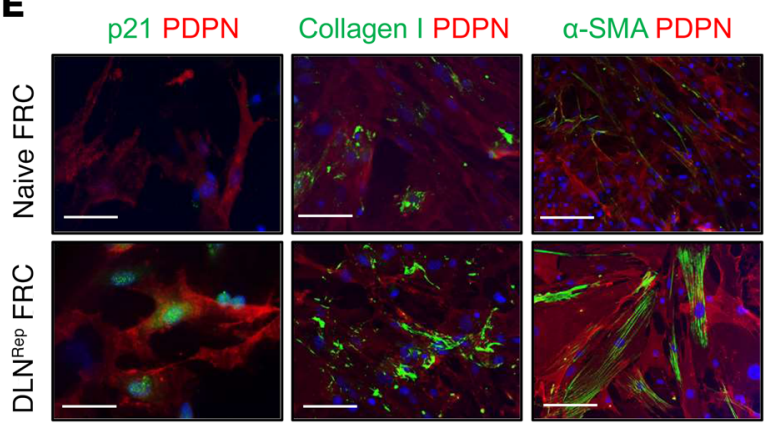

H


\section{K}

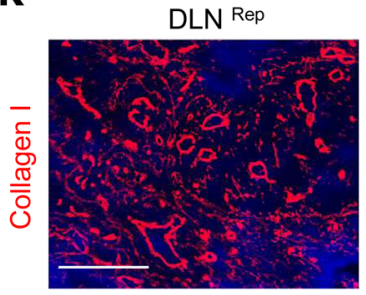

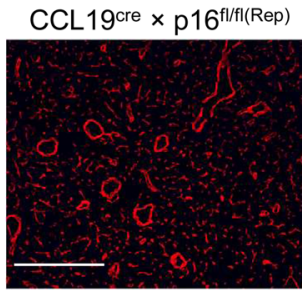



$\mathbf{F}$

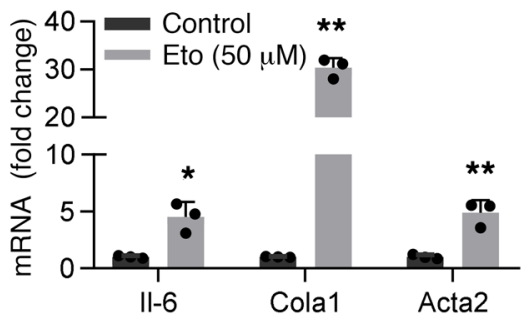

I

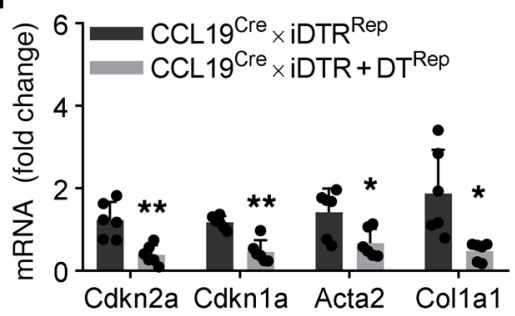

$\mathbf{L}$

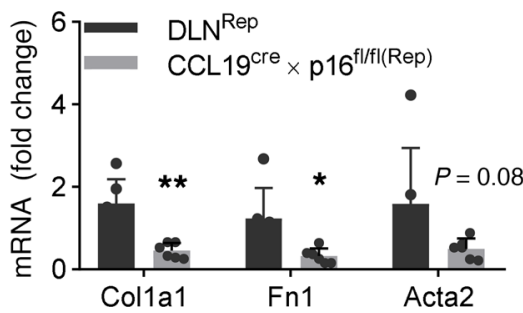

Figure 3. Senescent FRCs play a crucial role in the accumulation of ECM following transplantation. (A) Representative images of $\beta$-gal (senescence marker) staining in DLN ${ }^{R e p}$ and a LN of an age-matched naive mouse. Scale bars: $50 \mu \mathrm{m}$. (B) Costaining of PDPN ${ }^{+}$FRCs (red) and the senescence marker p21 ${ }^{\text {Wafi/cip1 }}$ (p21) in sections of DLN Rep and an age-matched naive LN. Scale bars: $50 \mu \mathrm{m}$. (C) Expression levels of senescence genes in DLN ${ }^{\text {Rep }}$ and an age-matched naive LN. $n=4$ (each dot represents 1 sample). (D) Staining with $\beta$-gal and (E) costaining with PDPN (red) for p21, collagen I, and $\alpha$-SMA in FRCs from a naive LN and DLNRep. Scale bars: $50 \mu \mathrm{m}$. (F) Gene expression levels of $/ / 6$, Col1a1, and Acta2 in FRCs following in vitro treatment with the senescence inducer etoposide (Eto). (C) Schematic of the generation of CCL19 ${ }^{\text {cre }}$ iDTR mice. (H) Collagen I+ fibers (red) in the DLNs of CCL19 ${ }^{\text {cre }}$ iDTR allogeneic skin transplant recipient mice

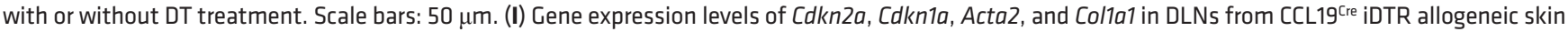
transplant recipient mice with or without DT treatment $(n=6)$. (J) Schematic of the generation of CCL19 $9^{\text {Cre }}$ p16 $6^{f / / f l}$ mice with FRC conditional knockout of p16. (K) Collagen I fibers (red) in DLNs of C57BL/6] and CCL19 $9^{\text {(re }}$ p16 $6^{\mathrm{fl} / \mathrm{fl}}$ recipient mice following allogeneic repetitive skin transplantation. Scale bars: $50 \mu \mathrm{m}$. (L) Gene expression levels of Col1a1, Fn1, and Acta2 in DLNs $(n=6)$. The percentage of areas stained positive in the fluorescence micrographs was assessed in 3-6 random microscopic fields for each mouse. Data are presented as the mean \pm SD. ${ }^{*} P<0.05$ and ${ }^{* *} P<0.01$, by Student's $t$ test.

tein marker $\mathrm{p} 21$, along with collagen I and $\alpha$-SMA, more robustly than did FRCs isolated from the naive LNs $(P<0.01$, Figure $3 \mathrm{E}$ and Supplemental Figure 3D). Next, we assessed whether treatment of FRCs with etoposide (a senescence-inducing agent) ex vivo would increase the expression of ECM markers such as collagen I in a similar manner. We found that ECM genes and Il6, the key cytokine linked to the SASP, were significantly upregulated in FRCs treated with etoposide compared with expression of these genes in untreated FRCs $(P<0.05$, Figure $3 F)$. In addition, IF staining of etoposide-treated FRCs revealed higher expression of p16, p21, $\alpha$-SMA, and collagen I as compared with expression levels in untreated FRCs (Supplemental Figure 3E).

Next, we sought to determine the effect of FRC depletion on the production of ECM in DLNs following allogeneic skin trans- 
A



\section{FRC}

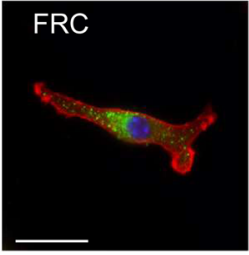

\section{B}

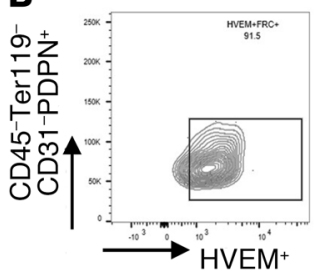

C DLN Rep

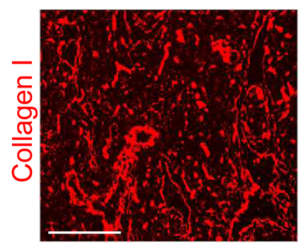

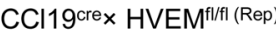

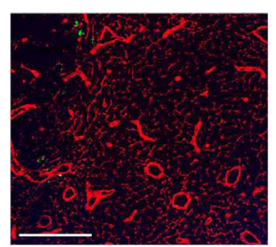

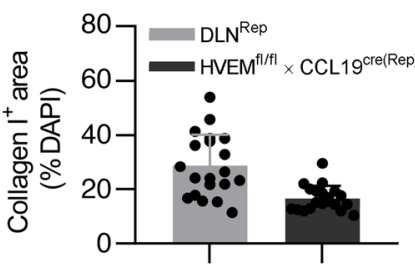

D
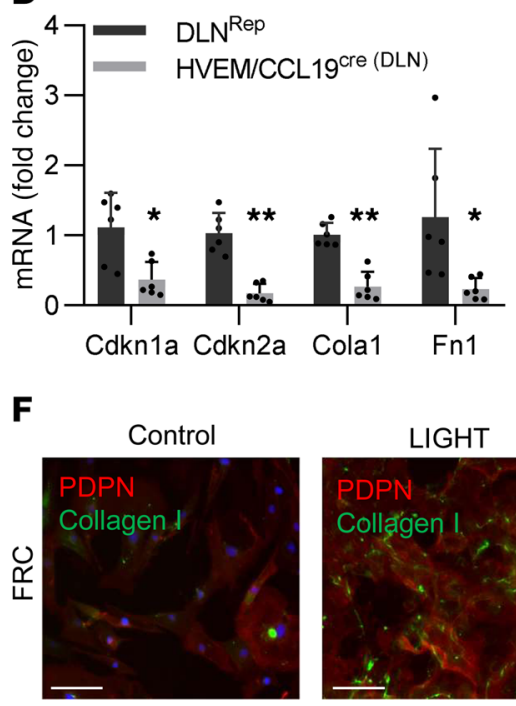

E

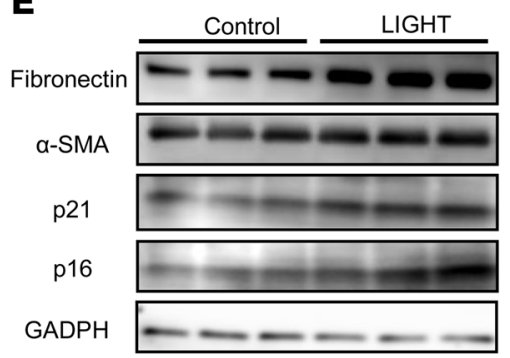

G

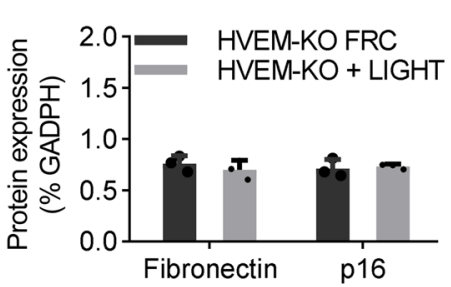



H



Figure 4. LIGHT increases ECM accumulation in DLNs by binding to HVEM in FRCs following transplantation. (A) IF staining for HVEM (green) expression in PDPN ${ }^{+}$FRCs (red) in vitro. Scale bar: $10 \mu \mathrm{m}$. (B) Flow cytometric analysis of HVEM expression by FRCs through gating on the CD45-CD31-PDPN ${ }^{+}$cell

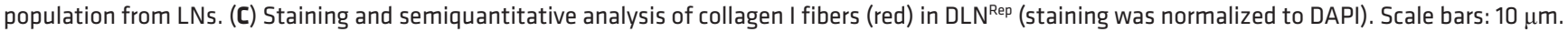
$n=4$. (D) Gene expression levels of senescence and fibrosis genes in FRCs from WT and HVEM-KO mice treated with LICHT (25 ng/mL). $n=6$. (E) Western blot and quantification of protein levels of fibronectin, $\alpha$-SMA, p21, and p16 in cultured WT FRCs after treatment with LIGHT (25 ng/mL). (F) IF staining of cultured WT FRCs with collagen I (green) and PDPN (red) after LIGHT ( $25 \mathrm{ng} / \mathrm{mL}$ ) treatment compared with no treatment. Scale bars: $10 \mu \mathrm{m}$. (G) Measurement of protein levels of fibronectin and p16 in cultured HVEM-KO FRCs after LIGHT stimulation. (H) IF staining for LIGHT expression in mast cells. Scale bar:10 $\mu \mathrm{m}$. The percentage of areas stained positive in the fluorescence micrographs was assessed in 3-6 random microscopic fields for each mouse. Data are presented as the mean $\pm \mathrm{SD}$. ${ }^{*} P<0.05$ and ${ }^{* *} P<0.01$, by Student's $t$ test.

plantation through the use of transgenic CCL19 ${ }^{\text {Cre }}$ inducible diphtheria toxin receptor (iDTR) mice as transplant recipients (Figure $3 G)$. We found that the density of collagen I fibers in the DLNs was markedly lower following FRC depletion (by DT before transplantation) as compared with those in CCL19 ${ }^{\text {Cre }}$ iDTR mice that did not receive DT $(P<0.01$, Figure $3 \mathrm{H}$ and Supplemental Figure $3 \mathrm{~F})$. In addition, the gene expression levels of Cdkn2a, Cdkn1a, Acta2, and Col1a1 were also significantly lower in the DLNs of FRCdepleted mice $(P<0.05$, Figure 3I).

Next, we generated CCL19 ${ }^{\text {Cre }} \mathrm{p} 16^{\mathrm{fl} / \mathrm{fl}}$ mice, in which the key senescence gene p16 is deficient specifically in FRCs, and we subjected these mice to repetitive skin transplantation (Figure 3J). $\mathrm{DLN}^{\text {Rep }}$ from these mice contained fewer collagen $\mathrm{I}^{+}$fibers $(P<$ 0.05 , Figure $3 \mathrm{~K}$ and Supplemental Figure $3 \mathrm{G}$ ) and had significantly lower levels of Colla1, Fn1, as well as Acta2 mRNA in comparison with control mice $(P<0.05$, Figure $3 \mathrm{~L})$. These data indicate that upregulation of senescence genes in FRCs plays a key role in the deposition of collagen I in DLNs after transplantation.

Expression of HVEM in FRCs and its impact on fibrosis in the DLN following allogeneic transplantation. Activities of the members of the TNF receptor (TNFR) superfamily, such as the LT $\beta$, are crucial to the formation of $\mathrm{LNs}$, as proven by $\mathrm{LT} \mathrm{R}^{-/-}$mice, which lack LNs. HVEM, another member of the TNFR superfamily, has not received similar attention. HVEM interacts with multiple ligands, including LIGHT. Interestingly, Croft's group showed that the interaction between LIGHT and HVEM contributes importantly to the promotion of both skin and pulmonary fibrosis $(26,27)$.

First, we found that our $\mathrm{PDPN}^{+}$cultured FRCs expressed HVEM (Figure 4A). By gating under the CD45-TER119-CD31$\mathrm{PDPN}^{+}$population of cells isolated from fresh LNs, we found by flow cytometry that up to $90 \%$ of FRCs expressed HVEM (Figure $4 \mathrm{~B}$ and Supplemental Figure $4 \mathrm{~A}$ ). An important question to answer was whether the absence of HVEM on FRCs would reduce the accumulation of collagen in the DLNs of mice that received repetitive transplants. Therefore, we generated a new conditional knockout mouse by breeding CCL19 ${ }^{\text {Cre }}$ mice with a $\mathrm{HVEM}^{\mathrm{fl} / \mathrm{fl}}$ strain (Supplemental Figure 4B). We confirmed that PDPN ${ }^{+}$FRCs in the LNs of these mice did not express HVEM (Supplemental Figure 4C). We performed repetitive skin transplantations in CCL19 Cre $\mathrm{HVEM}^{\mathrm{f} / \mathrm{fl}}$ recipients, in a manner similar to that described in the 
A

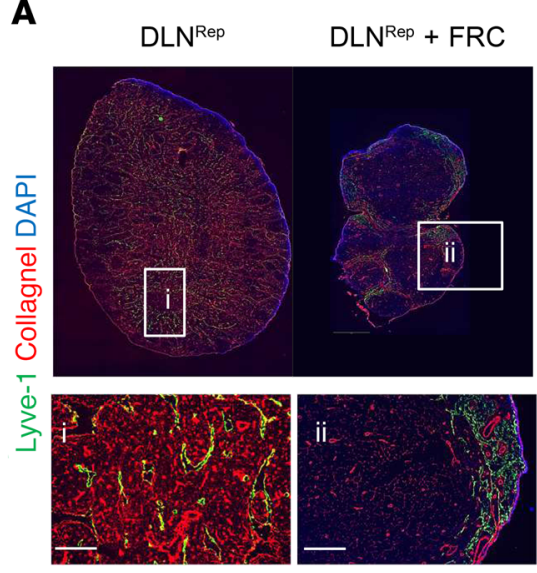

B

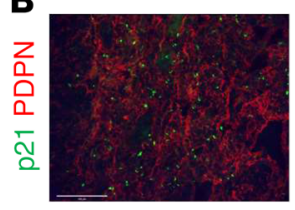

\section{C}

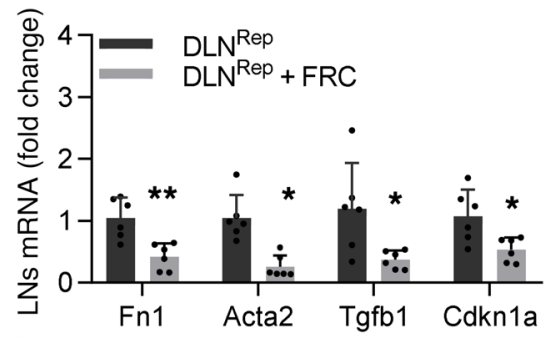

D

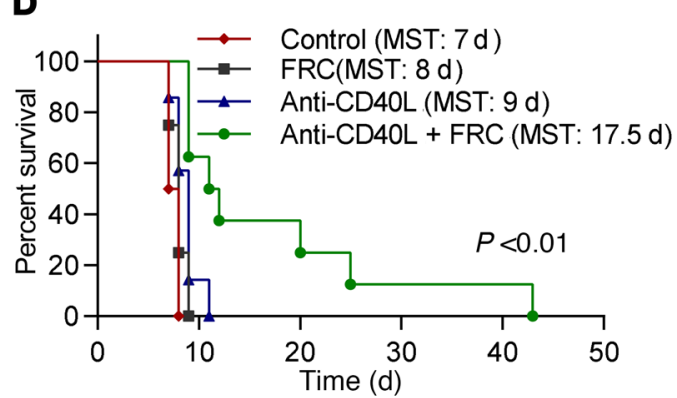

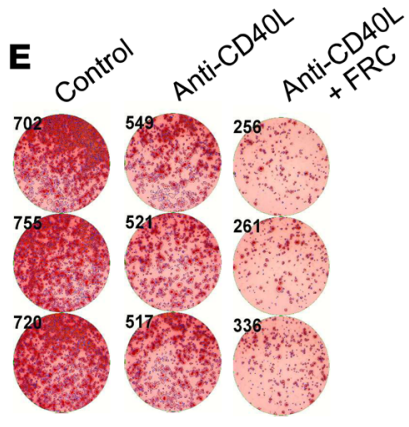

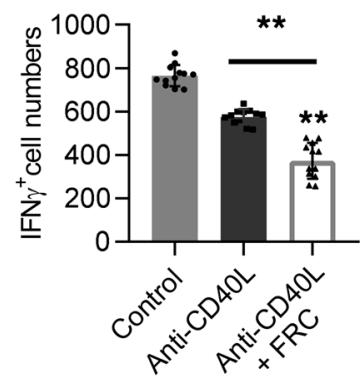

Figure 5. Injection of healthy FRCs decreases ECM accumulation induced by transplantation. (A) IF staining of collagen I fibers (red) in DLNRep and DLN Rep plus FRC mice. Scale bars: $1000 \mu \mathrm{m}$ and $50 \mu \mathrm{m}$ (enlarged insets). (B) Costaining of the senescence indicator p21 (green) with PDPN (red) in the DLN ${ }^{\text {Rep }}$ and DLN ${ }^{\text {Rep }}$ plus FRC treatment groups. Scale bars: $50 \mu \mathrm{m}$. (C) Gene expression levels of Fn1, Acta-2, Tgfb1, and Cdkn1a in the DLN ${ }^{\text {Rep }}$ of untreated mice and those treated with healthy FRCs $(n=6)$. (D) Kaplan-Meier survival curve for untreated mice (control: red, $n=4)$ and mice treated with FRCs $\left(5 \times 10^{4} /\right.$ mouse, black, $n=4)$, anti-CD4OL ( $9 \mu \mathrm{g} /$ mouse, blue, $n=7)$, and anti-CD4OL $\left(9 \mu \mathrm{g} /\right.$ mouse) plus FRCs $\left(5 \times 10^{4} /\right.$ mouse) (green, $\left.n=8\right)$ following allogeneic heart transplantation. MST, mean survival time. ${ }^{* *} P<0.01$, by log-rank (Mantel-Cox) test. (E) ELISpot assay shows the number of IFN- $\gamma^{+}$splenocytes in the untreated group, the anti-CD4OL treatment group, and the anti-CD4OL plus FRC treatment group on day 7 after heart transplantation $(n=4)$. The percentage of areas stained positive in the fluorescence micrographs was assessed in 3-6 random microscopic fields for each mouse. Data are presented as the mean \pm SD. ${ }^{*} P<0.05$ and ${ }^{* *} P<0.01$, by 2-tailed Student's $t$ test $(\mathbf{A}-\mathbf{C})$ and 2 -way ANOVA with Tukey's multiple comparisons post hoc test (E).

experiments above. The results indicated that collagen I deposition was markedly reduced in CCL19 ${ }^{\text {ree }} \mathrm{HVEM}^{\mathrm{I} / \mathrm{l}}$ mice in comparison with controls $(P<0.05$, Figure $4 \mathrm{C})$. The gene expression levels of the senescence markers Cdkn1a and Cdkn2a as well as Col1a1 and $F n 1$ were also lower in the DLN ${ }^{\text {Rep }}$ of CCL19 ${ }^{\text {Cre }} H_{V E M} M^{\mathrm{t} / \mathrm{l}}$ mice $(P<0.05$, Figure $4 \mathrm{D})$.

These findings indicated that stimulation of the HVEM pathway in FRCs plays an important role in their differentiation and production of ECM. Therefore, we sought to determine whether stimulation of FRCs with LIGHT would enhance their differentiation toward scar-forming myofibroblasts and promote adoption of the SASP. Treatment of FRCs with LIGHT markedly increased their expression of the ECM proteins fibronectin and $\alpha$-SMA as well as the senescence markers p16 and p21 $(P<0.05$, Figure 4E). IF staining also showed that these FRCs expressed collagen I more robustly (Figure 4F and Supplemental Figure 4D). Then, we isolated FRCs from the LNs of CCL19-Cre HVEM ${ }^{\mathrm{n} / \mathrm{l}}$ mice. Stimulation of these FRCs with LIGHT had no significant effect on the expression of fibronectin or the senescence marker p16 (Figure $4 \mathrm{G})$. These data provide evidence that LIGHT stimulates a phenotypic transition of FRCs into myofibroblasts. Furthermore, we found through IF staining that P815 cells, a mouse mast cell line, expressed LIGHT (Figure $4 \mathrm{H}$ ).

Delivery of healthy FRCs to the DLN Rep restores its microarchitecture and immunoregulatory function. We performed repetitive skin transplantations as described above. One group of mice received weekly i.v. injections of $5 \times 10^{4} \mathrm{FRCs}$. $\mathrm{DLN}^{\mathrm{Rep}}$ from the FRC-treated mice contained significantly less collagen I deposition and lower expression of the senescence marker p21 as compared with DLN ${ }^{\text {Rep }}$ from vehicle-treated mice (Figure 5, A and B, and Supplemental Figure 5A, respectively). In addition, we confirmed by quantitative real-time PCR (qRT-PCR) that the mRNA expression levels of Fn1, Acta-2, Tgfb1, and Cdkn1a were also lower in $\mathrm{DLN}^{\mathrm{Rep}}$ from FRC-treated mice $(P<0.05$, Figure 5C). Finally, IF staining for FoxP3 ${ }^{+}$Tregs in $\mathrm{DLN}^{\mathrm{Rep}}$ of mice in the FRC-treated group revealed a larger population of Tregs compared with that seen in DLN ${ }^{\text {Rep }}$ of mice from the untreated group $(P<0.05$, Supplemental Figure 5B).

Next, we tested whether treatment with FRCs inhibits acute rejection and improves the outcome of allogeneic heart transplantation with and without low-dose costimulatory blockade (using anti-CD4OL). We transplanted hearts from BALB/c donors into $\mathrm{C} 57 \mathrm{BL} / 6 \mathrm{~J}$ recipients and divided the mice into 2 groups: 1 group received anti-CD4OL (9 $\mu \mathrm{g} / \mathrm{mouse})$, and the other group received anti-CD4OL ( $9 \mu \mathrm{g}$ per mouse) and injections of $5 \times 10^{4}$ FRCs on the day of transplantation as well as $-1,1$, 3 , and 5 days after transplantation. We observed no difference in graft survival between control mice and mice in the FRCtreated group. However, FRC treatment synergized with antiCD4OL treatment and significantly prolonged graft survival compared with the groups that were treated with anti-CD4OL or FRCs alone $(P<0.01$, Figure 5D). 
A subset of transplanted mice was sacrificed on day 7 after transplantation. The DLNs (mediastinal LNs) of the anti-CD4OL plus FRC treatment group were markedly smaller and contained fewer expanded collagen I and lymphatic vessels than did DLNs of the control groups (Supplemental Figure 5C). Next, we performed an enzyme-linked immunospot (ELISpot) assay using splenocytes from each group and found that the IFN- $\gamma^{+}$cell population was highest in the control (untreated) group of transplant recipients in comparison with the group that received low-dose anti-CD4OL and the group that received anti-CD4OL plus FRCs. Importantly, FRC treatment was associated with a significantly lower IFN- $\gamma^{+}$ cell population in the spleens compared with mice that received anti-CD40L alone $(P<0.01$, Figure $5 \mathrm{E})$. This trend was confirmed by flow cytometry $(P<0.05$, Supplemental Figure $5 D)$. We labeled the FRCs with fluorescent 5-chloromethylfluorescein diacetate (CMFDA) dye and injected them into naive C57BL/6J mice to understand the cells' in vivo trafficking behavior. We detected CMFDA-labeled FRCs in the vicinity of the HEVs in the T cell zone of LNs 24 hours later, indicating that FRCs can home to LNs (Supplemental Figure 5E).

\section{Discussion}

LNs orchestrate a robust immune response following exposure to foreign antigens. LNs are also required for the resolution of ongoing immune responses, so they play different roles at various phases in the evolution of diverse immune responses (28). These multifaceted functions of LNs arise from their identity as extremely specialized organs with unique microvasculature, stromal fibers, and FRCs. FRCs provide the 3D scaffold that supports the LN microenvironment (29) and also, like other stromal cells, adapt the immune phenotype to each phase of the immune response (30-34). Despite the focus that immunologists have devoted previously to the effector immune responses that occur within the $\mathrm{LN}$, the cellular and molecular mechanisms that govern the microanatomical adaptation of the LN during immune activation or tolerance induction as well as development of therapeutic strategies that promote a regulatory LN microenvironment and result in immune tolerance have not received adequate attention.

Here, we show that early stimulation of FRCs by mast cells induced the production of collagen I, which is the prominent ECM fiber in the LN. This ECM is a prerequisite to the many microanatomical adaptions that are required to support an expansion of proliferating immune cells in the $\operatorname{LN}(5,10)$. Mast cells are recognized classically for their role in allergic reactions, but they are also important initiators of innate immunity (35). They possess a repertoire of vasoactive and proinflammatory cytokines than can tune the magnitude of the adaptive immune response (36-39). Strikingly, we found that mast cells accumulated in the DLN and that an absence of donor mast cells markedly reduced the deposition of collagen I in the DLN shortly following skin transplantation. Mast cells from C57BL/6-Tg (UBC-mCherry) donors migrated from the graft to the recipient DLN within 2 hours, where they comprised the vast majority of mast cell infiltrates. Since mast cells are rapidly activated, their activity is probably important for the formation of early post-transplantation inflammatory responses driven by mast cell degranulation. Thus, future testing of the efficacy of depleting mast cells in the allograft directly using a mast cell stabilizer in the perfusate before transplantation to improve allograft survival will be an important next step.

Another key finding here was the higher production of collagen I and lymphatic expansion in the DLNs of ischemic allografts. These observations set the stage for investigation of an entirely novel mechanism that may explain the increased risk of graft rejection associated with ischemic organs (40-45). Interestingly, exposure of mast cells to IRI potentiated the production of their secretory repertoire, which further stimulated the production of collagen I by FRCs and the expansion of lymphatics in the DLN. This expansion of lymphatic vasculature in the DLNs of ischemic skin allografts could be due to hypersecretion of VEGF by ischemic mast cells. Therefore, these findings indicate that donor mast cells activated by IRI could augment transplant immunity by activating FRCs and priming the LN microanatomically.

As transplant rejection occurs and transplant immunity subsides, we expected that the microarchitecture in the DLN would recover to its former resting state in a manner similar to that seen in other organs following IRI (e.g, kidneys). To our surprise, collagen I production persisted in the DLN, long after the rejection of the allografts. These data may suggest that the recovery of the stromal compartment of the DLN following excessive immune activation was not complete. To some extent, this lack of full recovery could be due to the lower frequency of stromal cells in the LN in comparison with other organs.

Next, we questioned whether the recovery of the stromal compartment of the DLN would be impaired further under continuous immune stimulation, such as in the case of repetitive skin transplantation in the same area. Tissue fibrosis is characterized by excessive deposition of ECM fibers containing collagen I and fibronectin (46). We noted a significant increase in both collagen I and fibronectin in the DLN ${ }^{\text {Rep }}$ compared with a naive LN. Notably, both collagen I and fibronectin were robustly deposited in a thick-

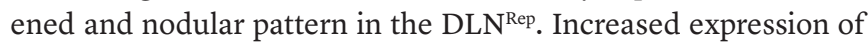
$\mathrm{PDPN}^{+} \alpha$-SMA ${ }^{+}$cells is highly suggestive of a potential transition of FRCs toward myofibroblasts (47). The DLN also stained positive for Masson's trichrome, a finding consistent with organized fibrosis. Electron micrographs of DLN ${ }^{\text {Rep }}$ showed marked accumulation of collagen I and mast cells. The early transformation of fibroblasts into protomyofibroblasts appears to depend on accumulation of ECM and activation of the TGF- $\beta$ signaling pathway (48, 49). We found that expression of TGF- $\beta$ pathway-related genes was higher in the DLN ${ }^{\text {Rep }}$ than in naive LNs. An important future study should investigate the mechanisms by which chronic mast cell accumulation and activation of the TGF- $\beta$ R on FRCs promote the development of fibrosis in DLN ${ }^{\text {Rep }}$.

Several FRC-tropic pathogens have been shown to impair LN stroma $(5,50,51)$. Interestingly, FRC preservation in SIV-infected animals normalized the $\mathrm{T}$ cell population in LNs (52). Multiple studies now indicate that the DLN undergoes scarring following a number of infectious challenges $(53,54)$. Using a renal IRI model, we have shown that repetitive injuries are also associated with KLN fibrosis (17).

In addition, this observation of fibrosis in the DLN following transplantation has an important implication in the current era of transplantation, in which many patients receive second and third 
transplants. Cellular senescence has been commonly associated with chronic inflammation following sustained exposure to stress inducers, such as proinflammatory cytokines (55-57). Senescent cells upregulate the cyclin-dependent kinase inhibitors p16, p53, and 21 to block cell replication $(58,59)$. The process of senescence also induces a number of additional phenotypic alterations in the cells, including an increased proinflammatory secretome referred to as the SASP $(60,61)$. The prolonged presence of senescent cells in a tissue can contribute significantly to tissue damage due to the persistence of their associated proinflammatory features (25). Our data indicate, importantly, that FRCs within the $\mathrm{DLN}^{\mathrm{Rep}}$ became senescent, and, to our knowledge, no information regarding the senescence of LN FRCs during alloimmunity currently exists. DLNs from recipient C57BL/6 mice following repetitive allogeneic skin transplantation had high expression levels of the gold-standard senescence marker $\beta$-gal as well as of the cell-cycle regulators p16, p21, p53, p57, as demonstrated by both qRT-PCR and IF staining. In addition, FRCs isolated from $\mathrm{DLN}^{\mathrm{Rep}}$ also expressed $\beta$-gal and p21 more robustly than did those from naive LNs. This finding was associated with elevated transcription of ECM markers (collagen I and $\alpha$-SMA) and SASP components such as IL-6.

An important question to answer was whether the absence of FRCs would reduce accumulation of collagen in the DLNs of mice that received repetitive transplants. CCL19 is expressed almost exclusively by FRCs, and CCL19 ${ }^{\text {Cre }}$ mice can be used to deplete some genes in an FRC-specific and spatiotemporally controlled manner through the use of Cre-iDTR and Cre-loxP recombination (51, 62). Therefore, we generated a new FRC conditional-knockout mouse by breeding CCL19 ${ }^{\text {Cre }}$ mice with an iDTR strain. We confirmed that the CCL19 ${ }^{\text {Cre }}$ iDTR mice had a depleted PDPN ${ }^{+}$FRC population (data not shown), and collagen I deposition as well as senescence-related DNA damage observed in the DLN ${ }^{\mathrm{Rep}}$ were markedly less severe in these mice. $16^{\mathrm{INK} 4 \mathrm{a}}$ is a key cell-cycle inhibitor that is upregulated in senescent cells (63). The CDKN2A locus, which is responsible for producing the cyclin-dependent kinase inhibitor $\mathrm{p} 16^{\mathrm{INK4a}}$ and lies dormant in normal replicating cells, becomes activated in most senescent cells (63). Next, we generated FRCspecific p16 ${ }^{\mathrm{INK} 4 \mathrm{a}}$ conditional-knockout mice on a C57BL/6 background (referred to herein as CCL19 ${ }^{\mathrm{Cre}} \mathrm{p} 16^{\mathrm{fl} / \mathrm{ll}}$ mice) in order to better understand the effect that deletion of $\mathrm{p} 16^{\mathrm{INK} 4 \mathrm{a}}$ activity in FRCs exerts on their phenotype. Our data showed that inhibition of p16 ${ }^{\text {INK4a }}$ in FRCs markedly reduced ECM deposition.

The LT $\beta$ R is a key member of the TNFR superfamily that governs the development of the LN (64). Our group has carried out several studies on this pathway and its importance to transplant immunity. Mice deficient for the LT $\beta$ R lack LNs $(17,65-67)$. There has been a paucity of studies of other equally important TNFRs, such as HVEM. Here, we show for the first time to our knowledge the presence of HVEM in FRCs and that stimulation of FRCs with LIGHT promoted their differentiation toward scar-forming myofibroblasts. Interestingly, LIGHT and HVEM may contribute importantly to promoting both skin and pulmonary fibrosis $(26,27)$. Future studies are required to assess how LIGHT production by a wide variety of immune cells, including mast cells, contributes to propagation of the proinflammatory immune response in the DLN $(68,69)$.

FRCs share some phenotypic features of mesenchymal stromal cells (MSCs), which have been tested as a potential therapy for several diseases (70). These characteristics include their fibroblastic appearance and their stromal cell-surface markers. However, at the transcriptomic levels, FRCs differ from other stromal populations, as FRCs contain a high degree of polarization for immune-enriched genes (71). Our observation that FRCs adopt a SASP resembling myofibroblasts as the DLN becomes fibrotic led us to investigate whether systemic administration of FRCs that were expanded ex vivo would restore the microarchitecture of the DLN and contribute to transplant tolerance. Indeed, we found that treatment with healthy FRCs prolonged survival in murine cardiac allograft recipients and was associated with antiinflammatory features in the DLN, including restoration of its native microarchitecture and a higher population of Tregs. Our group has shown recently that gut microbiota regulate transplant immunity via $\mathrm{LN}$ remodeling (72). Others have shown that treatment with ex vivo-expanded FRCs reduces sepsis-related mortality (73). We have also shown that FRC administration can remodel LN microarchitecture and reduce kidney ischemic injuries (17). Altogether, these data highlight the potential importance of how LN stromal remodeling can impart a broad range of medical applications. Finally, FRCs express CD40, and anti-CD40L may prevent immunostimulatory interactions of FRCs with T cells, as demonstrated previously (66). Therefore, addition of anti-CD4OL may inhibit the proinflammatory capacity of FRCs in the DLN directly.

Future experiments are required to further decipher the relative contributions of inflammatory responses and fibrosis in the LN to the promotion of transplant rejection. One potential approach would be to attempt to inhibit collagen synthesis in the LN through targeted delivery of an antibody against LIGHT and assess its effects in promoting tolerance. We have already established the efficacy of a nanoparticle-based method of delivering therapeutic agents to activated LNs (74) that we can use in this experiment. We are also interested in more closely examining the molecular mechanisms behind the potential antiinflammatory effects of exogenous FRCs in prolonging allograft survival, especially those that occur as a result of FRC interactions with donor-derived mast cells. In this study, the immunosuppressive impact of multiple injections of FRCs likely overwhelmed the initial proinflammatory activity of the donor-derived mast cells in the DLNs.

Here, we illuminate critical signaling pathways that impact the function of stromal cells in the LN and thereby modify its immunological microenvironment (Figure 6). We believe these studies will not only advance our knowledge but will also identify entirely new targets and approaches to establish highly innovative immune therapies that promote a regulatory microenvironment in the LN following transplantation.

\section{Methods}

Mice. The C57BL/6J (JAX no. 000664); BALB/cByJ (JAX no.

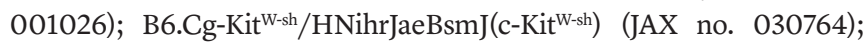
B6.129S7-Rag1 $1^{\left.\text {tm1Mom} / J ~ （ R a g 1^{-/}\right)}$(JAX no. 002216)，B6(Cg)-Tyr ${ }^{c-2 J}$ Tg(UBC-mCherry)1Phbs/J; and C57BL/6-Gt (ROSA)26Sor ${ }^{\text {tm1 (HBEGF)Awai/J }}$ (C57BL/6-iDTR, referred to herein as iDTR) mice used in these experiments were purchased from The Jackson Laboratory. CCL19 ${ }^{\text {Cre }}$ mice were a gift from Shannon Turley at Genentech (South San Francisco, California, USA). HVEM ${ }^{\mathrm{f} / \mathrm{l}}$ mice, which have loxP sites flanking exons 
Pro-inflammatory microenvironment of DLN following repetitive transplantation

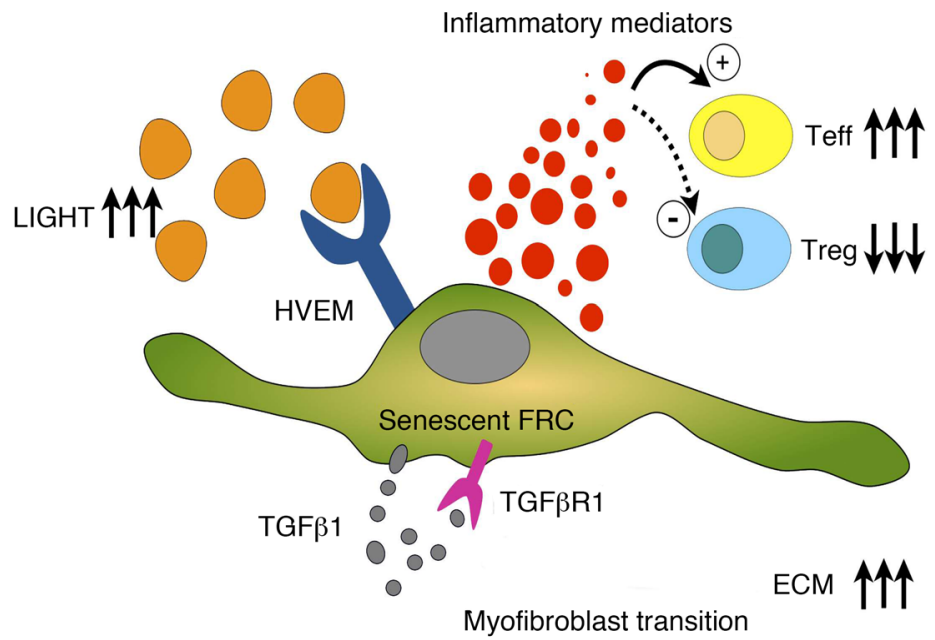

Figure 6. Schematic presentation of a senescent FRC in the proinflammatory microenvironment of the DLN following repetitive transplantation. Sustained stimulation of HVEM by LICHT on the surface of the FRC results in increased production of ECM and its phenotypic transformation toward a myofibroblast, creating and nurturing an immunostimulatory milieu in the LN. Teff, effector T cell.

3 and 6 of the Tnfrsf 14 gene, were obtained from Mitchell Kronenberg (La Jolla Institute for Allergy and Immunology, La Jolla, California, USA). Floxed p16 mice (p16 ${ }^{\mathrm{L}}$, referred to herein as $\mathrm{p} 16^{\mathrm{fl} / \mathrm{ll}}$ mice) were obtained from David Darr (University of North Carolina, Chapel Hill, North Carolina, USA). CCL19 ${ }^{\text {Cre }}$ mice were backcrossed with C57BL/6iDTR, HVEM ${ }^{\mathrm{t} / \mathrm{l}}$, and $\mathrm{p} 16^{\mathrm{t} / \mathrm{ll}}$ mice, generating CCL19 ${ }^{\mathrm{Cre}}$ iDTR, CCL19 ${ }^{\mathrm{Cre}}$ $\mathrm{HVEM}^{\mathrm{f} / \mathrm{fl}}$, and CCL19 ${ }^{\mathrm{Cre}} \mathrm{p} 16^{\mathrm{fl} / \mathrm{fl}}$ mice, respectively. Offspring were genotyped by PCR, according to the protocol from The Jackson Laboratory. Male and female mice aged 6-10 weeks (20-25 g) were used in all experiments. The mice were housed in standard laboratory conditions and were fed a normal diet in a pathogen-free animal facility. Three to 6 mice per group were used in each individual experiment.

Mouse skin transplantation. The allogeneic skin transplantation model was used for all experiments. Briefly, full-thickness trunk skin was harvested from donors and cut into a $1-\mathrm{cm}^{2}$ graft. The full-thickness skin graft was excised from the right side of the donor mice under anesthesia with isoflurane, and the graft was sutured with 6-0 silk onto flank wounds produced on the recipient mice. Bandages were used to secure the skin grafts for 7 days. Age-matched naive mice were used as controls. DLNs were harvested at the indicated time points. For the ischemic skin transplant model, the donor graft was kept in PBS for 8 hours at $4^{\circ} \mathrm{C}$, before its implantation in the recipient mice. Skin transplantation in the repetitive transplantation model was performed 3 times at 30-day intervals. Age-matched naive mice were used as controls. DLNs were harvested 30 days after the third transplantation.

Mouse heart transplantation. Vascularized intra-abdominal heterotopic transplantation of heart allografts was performed using microsurgical techniques, as described previously (75). Briefly, donor mice were infused with $1 \mathrm{~mL}$ cold heparin (BD Vacutainer Sodium Heparin, no. 366480 143USP units/10 mL) into the inferior vena cava (IVC). Following ligation of the superior vena cava and dissection of the IVC, the ascending aorta and pulmonary artery were tied, and the heart was harvested by cutting the blood vessels distal to the ties. Then, the donor hearts were stored at $4^{\circ} \mathrm{C}$ in sterile saline until transplantation. The hair overlying the abdomens of the recipient mice was shaved, and an incision was made over the midline, exposing the infrarenal aorta. The IVC and abdominal aorta were divided and clamped. The donor heart was brought to the recipient site, and the ascending aorta and pulmonary artery of the donor were attached to the abdominal aorta and IVC of the recipient mouse, respectively, using 10-0 sutures. The beating of the donor heart grafts was verified following release of the cross-clamp, and the abdominal wound was closed. A proportion of the heart transplantation mice were used to measure the survival curve. One day after transplantation, the heartbeat of the recipients was confirmed to assess graft survival. Complete rejection of the graft was defined as the absence of a heartbeat and confirmed by histology. On day 7 , five surgical mice were scarified, and the DLNs were removed for use in the experiments. Heart transplantations for the repetitive heart transplant model were performed twice, 30 days apart. DLNs were harvested 30 days after the second transplantation.

FRC depletion. Eight CCL19 ${ }^{\text {Cre }}$ iDTR mice were randomly divided into 2 groups of 4 mice each. Repetitive skin transplantation was performed in all mice. One group was injected i.p. with $100 \mu \mathrm{g}$ diphtheria toxin (DT) from Corynebacterium diphtheriae (MilliporeSigma) daily for 4 days, starting on the day of skin transplantation (days 0-4).

Toluidine blue staining of mast cells. Frozen sections of ileum (10- $\mu \mathrm{m}$-thick) were prepared and stained with toluidine blue, as described previously (76). A blinded assessment of 5 random fields from each section was performed at $\times 400$ magnification. Three mice were measured in each group. The total number of toluidine bluepositive cells was recorded.

IF staining. The examined organs were harvested and frozen in OCT blocks immediately at the designated time points. Frozen sections (8- $\mu$ m-thick) were cut using a cryostat. Fresh samples were fixed in cold acetone for 5 minutes, washed with PBS for 5 minutes, and blocked with $3 \%$ BSA solution. Then, the samples were stained with primary antibodies overnight at $4^{\circ} \mathrm{C}$ and with secondary antibodies for 30 minutes at room temperature. DAPI (VECTASHIELD, Vector Laboratories) was used to stain the nuclei. FRCs were prepared for staining first by seeding the cells in a sterile chamber slide (Lab-Tek, Thermo Fisher Scientific), before following the same steps described above. The primary antibodies included goat anti-mouse PDPN (R\&D Systems, AF3244); rabbit anti-mouse Lyve-1 (Abcam, ab14917; 1:300); rat anti-mouse CD11b (BioLegend, 101201; 1:100); rat anti-mouse MECA79 (Novus Biologicals, NB100-77673; 1:200); rabbit anti-mouse collagen I (Novus Biologicals, NB600-408; 1:200); rabbit anti-mouse fibronectin (Abcam, ab2413; 1:300); rat anti-mouse ERTR-7 (Santa Cruz Biotechnology, sc73355); rabbit anti-mouse $\alpha$-SMA (Cell Signaling Technology, 19245, 1:200), rabbit anti-mouse p21 $1_{\text {Waf1/Cip1 }}$ (Cell Signaling Technology, 2947, 1:200); and rabbit antimouse CDKN2A/p16 ${ }^{\text {INK4a }}$ (Abcam, ab2D9A12, 1:100). The secondary antibodies included donkey anti-goat 594 IgG (Jackson ImmunoResearch, no. 705-587-003, 1:200); donkey anti-rat IgG 488 (Jackson ImmunoResearch, no. 712-545-150,1:200); donkey anti-rabbit 594 IgG (Jackson ImmunoResearch, no. 711-585-152, 1:200); donkey anti-rat 594 IgG (Jackson ImmunoResearch, no. 712-585-153, 1:200); 
donkey anti-rabbit 488 IgG (Jackson ImmunoResearch, no. 711-545152, 1:200); and goat anti-rat 488 IgM (Jackson ImmunoResearch, no. 112-547-020, 1:200). All images were captured using an EVOS FL Auto 2 Imaging System (Thermo Fisher Scientific). DAPI was used to stain the cell nuclei. For each sample, 5-6 random fields were used for semiquantitative analysis, and Image J was used to measure the positively stained area and to quantify cells.

Culture of primary FRCs. FRCs were isolated and purified as described previously (17). LNs were pooled from mice and digested in a mix containing collagenase P $(0.2 \mathrm{mg} / \mathrm{mL}$, Roche Diagnostics $)$ and dispase II $(0.8 \mathrm{mg} / \mathrm{mL}$, MilliporeSigma $)$ in a water bath at $37^{\circ} \mathrm{C}$ for 15 minutes. This step was repeated for multiple rounds, until all of the tissue was digested into a single-cell suspension. All digested cells were kept in complete DMEM containing 10\% FBS (Lonza) and cultured in flasks following isolation by centrifugation. Only cells from passages 4-6 were used in the experiments. The purity of FRCs (CD $45^{-}$CD 31 $\mathrm{PDPN}^{+}$) was assessed using flow cytometry.

Cell culture. P815 cells are a mast cell line that is derived from mastocytoma (ATCC). These cultured cells were maintained with DMEM containing $10 \% \mathrm{FBS}$ at $37^{\circ} \mathrm{C}$ in a $5 \% \mathrm{CO}_{2}$ humidified atmosphere. For the ischemia assay, P815 cells $\left(1 \times 10^{6}\right.$ cells $)$ were treated with $500 \mu \mathrm{M} \mathrm{H}_{2} \mathrm{O}_{2}$ for 30 minutes before being cultured in normal DMEM for an additional 24 hours.

Coculture of FRCs and P815 cells. FRCs $\left(1 \times 10^{5}\right.$ cells $)$ were seeded in 24-well plates. After 24 hours, the FRCs were treated with conditioned media from P815 cells with or without $\mathrm{H}_{2} \mathrm{O}_{2}$ stimulation for 48 hours. Then, the conditioned cells were incubated for 24 hours and collected for gene expression and staining experiments.

SVEC4-10 tube formation assay. SVEC4-10 is an endothelial cell line isolated from mouse axillary LNs; we obtained these cells as a gift from Jonathan Bromberg's laboratory (University of Maryland, Baltimore, Maryland, USA. Prechilled 48-well plates were coated with 200 $\mu \mathrm{L}$ Matrigel per well (BD Biosciences, 354234) and incubated at $37^{\circ} \mathrm{C}$ for 30 minutes. SVEC4-10 cells $\left(1 \times 10^{5}\right)$ were resuspended in conditioned medium from $\mathrm{P} 815$ cells with or without $\mathrm{H}_{2} \mathrm{O}_{2}$ stimulation for 48 hours and seeded onto the Matrigel layer. Following incubation for 24 hours, media were carefully aspirated from the wells to avoid disrupting the tube network on the basement membrane extract (BME), and each well was washed with $500 \mu \mathrm{L}$ Dulbecco's PBS (DPBS) (Corning). CellTracker Green CMFDA Dye (Thermo Fisher Scientific) was used to visualize the endothelial cell network. Five images per well were captured using an EVOS FL Auto 2 Imaging System (×20 magnification). Total network length, node numbers, and mesh numbers were assessed using the Angiogenesis Analyzer plugin (Carpentier G, 2012) in Image J. Data are represented as the mean of 3 random fields.

Recombinant LIGHT stimulation. FRCs was harvested and resuspended in 6-well plates $\left(5 \times 10^{5}\right.$ cells). After 24 hours, mouse recombinant LIGHT (TNFSF14, BioLegend, no. 557604) was dissolved in sterile PBS and used at a final concentration of $25 \mathrm{ng} / \mathrm{mL}$. The FRCs were collected 14 days after stimulation.

Adoptive transfer of FRCs. We performed repetitive skin allograft transplantation, and divided the mice into 2 groups: untreated (DLN ${ }^{\text {Rep})}$ and FRC-treated (DLN ${ }^{\text {Rep }}$ plus FRC). FRCs were washed with PBS once and harvested using 0.25\% trypsin-EDTA (Gibco, Thermo Fisher Scientific). Mice in the DLN ${ }^{\text {Rep }}$ plus FRC group were injected i.v. with 5 $\times 10^{4}$ FRCs once a week for a total of 10 weeks following the first skin transplantation. DLNs were harvested 30 days after the third trans- plantation. Heart allografts from BALB/c donors were transplanted into C57BL/6J recipients, and then the recipients were into 4 groups: no treatment (control), FRC treatment, anti-CD4OL treatment, and anti-CD4OL plus FRC treatment ( $n=4$ mice/group). Mice were injected i.v. with $5 \times 10^{4} \mathrm{FRCs}$ on days $-1,1,3$, and 5 . The anti-CD4OL treatment groups received anti-CD4OL ( $9 \mathrm{ng} /$ mouse) on the same schedule as the FRC treatment groups. The survival duration of the grafts was recorded during the experiment. For flow cytometric analysis and ELISpot assays, mice receiving heart allografts were divided into 3 groups: no treatment (control), anti-CD4OL treatment, and anti-CD4OL plus FRC treatment ( $n=4$ mice/group). The mice were sacrificed on day 7 , and the spleens were harvested for subsequent experiments. For the FRC trafficking test, we used the CMFDA dye to label the FRCs and i.v. injected $5 \times 10^{4} \mathrm{FRCs}$ into naive C57BL/6J mice. Then, after 24 hours, we harvested the DLNs and examined them for GFP signal.

Flow cytometry. Flow cytometric analysis of splenocytes harvested from mice in the untreated control group, anti-CD40L group, and antiCD4OL plus FRC group was performed, and each leukocyte population was quantified. The spleen was crushed and collected in a $50-\mathrm{mL}$ centrifuge tube. The resulting single-cell suspension was centrifuged at $350 \mathrm{~g}$ for 6 minutes. Then, ammonium chloride potassium (ACK) lysis buffer (Lonza) was used to lyse the red blood cells for 2 minutes. The cell suspension was centrifuged at $350 \mathrm{~g}$ for 6 minutes to remove the lysed cells. The pellet was resuspended in complete DMEM and seeded in a 96-well plate at a concentration of $1 \times 10^{6}$ cells/well. For intracellular cytokine staining, the splenocytes were first incubated with phorbol 12-mystirate 13-acetate (100 ng/mL) (MilliporeSigma), ionomycin $(1 \mathrm{mg} / \mathrm{mL}$ ) (MilliporeSigma), and GolgiStop protein transport inhibitor (BD Biosciences) at $37^{\circ} \mathrm{C}$ for 4 hours. Next, these cells were washed and stained according to standard protocols for intracellular marker staining. Flow cytometry was performed using the BD FACSCanto II flow cytometer (BD Biosciences). All antibodies were purchased from BD. Data were analyzed with FlowJo software.

$I F N-\gamma$ ELISpot assays. Splenocytes from WT BALB/c mice were used as stimulators, and splenocytes from C57BL/6 recipients of $\mathrm{BALB} / \mathrm{c}$ heart allografts with or without FRC and anti-CD4OL treatment were used as responders, as described previously (77). Briefly, stimulator cells were irradiated and added to responder cells at a 1:1 ratio on Millipore Immunospot plates (MilliporeSigma). Biotinylated detection antibodies were added to the wells and incubated according to the Mouse IFN- $\gamma$ ELISPOT Kit instructions (BD Biosciences). The Immunospot analyzer (Cellular Technology Ltd.) was used to read and analyze the plates.

Senescence-associated $\beta$-gal staining. Frozen DLN sections were used to stain $\beta$-gal according to the manufacturer's protocol (Senescence $\beta$-Galactosidase Staining Kit, Cell Signaling Technology, cata$\log$ 9860). Cultured FRCs were seeded in sterile chamber slides and allowed to attach to coverslips 1 day before staining. The staining protocol followed the instruction manual provided in the kit.

Electron microscopy. DLNs were fixed in Karnovsky's fixative solution. Samples were cut into approximately 1.5 -mm-thick pieces, subjected to a secondary fixative for 1 hour in $1 \%$ osmium tetroxide/ $0.3 \%$ potassium ferrocyanide in cacodylate buffer on ice, and stained for 1 hour with $2 \%$ aqueous uranyl acetate. Samples were cured in an oven at $60^{\circ} \mathrm{C}$ for 48 hours. Sections (70-nm-thick) were then taken and imaged on an FE-SEM (Zeiss Crossbeam 540) using the aSTEM detector. 
qRT-PCR experiments. Tissue samples for qRT-PCR were stored at $-80^{\circ} \mathrm{C}$ following harvesting from the mice. RNA from tissues and cultured cells was isolated following the standard approach using the Direct-zol RNA MiniPrep Plus kit (Zymo Research). Total RNA (100 ng) was reverse transcribed with a High-Capacity cDNA Reverse Transcription Kit (Applied Biosystems). qRT-PCR was carried out using iQ-SYBR Green Supermix (Bio-Rad) and the Bio-Rad CFX96 Real-Time System with the C1000 Touch Thermal Cycler, and all the reactions were performed in duplicate. Cycling conditions were $95^{\circ} \mathrm{C}$ for 3 minutes, 40 cycles of $95^{\circ} \mathrm{C}$ for 15 seconds, $60^{\circ} \mathrm{C}$ for 1 minute, and 1 cycle of $95^{\circ} \mathrm{C}$ for 10 seconds. Data were analyzed using the $2^{-\Delta \Delta \mathrm{Ct}}$ method via GADPH normalization. The primer sequences used are listed in Supplemental Table 1.

Western blot analysis. FRCs were harvested and lysed with RIPA buffer (MilliporeSigma). Proteins were separated by $4 \%-15 \%$ Mine Protean SDS-PAGE (Bio-Rad) and transferred onto PVDF membranes (MilliporeSigma). The membranes were blocked in 5\% skim milk in TBST (0.1\% Tween-20) solution for 30 minutes at room temperature. Membranes were incubated with primary antibodies at $4^{\circ} \mathrm{C}$ overnight and subsequently with an HRP-conjugated secondary antibody. The electrochemiluminescence detection solution was used to determine protein levels. Band intensity was quantified using ImageJ software $(\mathrm{NIH})$. The relative expression level was normalized to GADPH. We used the following primary antibodies: mouse fibronectin (Abcam, ab2413; 1:1000); rabbit anti-mouse $\alpha$-SMA (Cell Signaling Technology, 19245, 1:1000); rabbit anti-mouse p21 ${ }^{\text {wafl/Cip1 }}$ (Cell Signaling Technology, 2947, 1:1000); rabbit anti-mouse CDKN2A/p16 ${ }^{\text {INK4a }}$ (Abcam, ab2D9A12, 1:1000); and mouse anti-mouse GADPH (Santa Cruz Biotechnology, sc-32233, 1:100). We used HRP goat anti-mouse (Abcam, ab2D9A12, 1:3000); HRP goat anti-rabbit (Abcam, ab6721, 1:3000); and HRP goat anti-rat (Abcam, ab97057, 1:3000) secondary antibodies.
Statistics. Data were analyzed using an unpaired 2-tailed Student's $t$ test or a 2-way ANOVA with Tukey's multiple comparisons test for comparisons between experimental groups. For Kaplan-Meier survival graphs, a log-rank comparison of the groups was used to calculate the $P$ values. A $P$ value of less than 0.05 was considered significant. GraphPad Prism (GraphPad Software) was used for data analysis and to prepare the graphs. Data represent the mean \pm SD

Study approval. All animal procedures were performed in accordance with the relevant guidelines and regulations approved by the IACUC of Brigham and Women's Hospital (protocol 0167).

\section{Author contributions}

$\mathrm{XL}$ designed and performed experiments, analyzed data, and wrote the main text of the manuscript. JZ and MU performed microsurgery. VK revised the main text of the manuscript. NB performed ELISpot assays and analyzed data. LJ and TI revised the manuscript. MMM, DRL, JSB, SRS, and CFW critically reviewed the manuscript. RA designed the study, interpreted and analyzed data, and critically revised and finalized the manuscript. All authors edited and approved the manuscript.

\section{Acknowledgments}

This work was supported by grants from the NIH (R01AI126596, R01HL145813, and R01HL141815, to RA and R01AI062765 and R01AI14496, to JB).

Address correspondence to: Reza Abdi, Transplantation Research Center, Brigham and Women's Hospital, 221 Longwood Avenue, Boston Massachusetts 02116, USA. Phone: 617.732.5259; Email: rabdi@rics.bwh.harvard.edu.
1. Fletcher AL, et al. Reproducible isolation of lymph node stromal cells reveals site-dependent differences in fibroblastic reticular cells. Front Immunol. 2011;2:35.

2. Hammerschmidt SI, et al. Stromal mesenteric lymph node cells are essential for the generation of gut-homing T cells in vivo. J Exp Med. 2008;205(11):2483-2490.

3. Cording $\mathrm{S}$, et al. The intestinal micro-environment imprints stromal cells to promote efficient Treg induction in gut-draining lymph nodes. Mucosal Immunol. 2014;7(2):359-368.

4. Bénézech $C$, et al. Lymphotoxin- $\beta$ receptor signaling through NF- $\mathrm{B} 2$ 2-RelB pathway reprograms adipocyte precursors as lymph node stromal cells. Immunity. 2012;37(4):721-734.

5. Fletcher AL, Acton SE, Knoblich K. Lymph node fibroblastic reticular cells in health and disease. Nat Rev Immunol. 2015;15(6):350-361.

6. Marino J, Paster J, Benichou G. Allorecognition by $\mathrm{T}$ lymphocytes and allograft rejection. Front Immunol. 2016;7:582.

7. Boisgérault F, Liu Y, Anosova N, Dana R, Benichou G. Differential roles of direct and indirect allorecognition pathways in the rejection of skin and corneal transplants. Transplantation. 2009;87(1):16-23.

8. Buechler MB, Turley SJ. A short field guide to fibroblast function in immunity. Semin Immunol. 2018;35:48-58.
9. Brown FD, Turley SJ. Fibroblastic reticular cells: organization and regulation of the T lymphocyte life cycle. J Immunol. 2015;194(4):1389-1394.

10. Fletcher AL, Malhotra D, Turley SJ. Lymph node stroma broaden the peripheral tolerance paradigm. Trends Immunol. 2011;32(1):12-18.

11. Astarita JL, et al. The CLEC-2-podoplanin axis controls the contractility of fibroblastic reticular cells and lymph node microarchitecture. $\mathrm{Nat}$ Immunol. 2015;16(1):75-84.

12. Lu TT, Browning JL. Role of the lymphotoxin/ LIGHT system in the development and maintenance of reticular networks and vasculature in lymphoid tissues. Front Immunol. 2014;5:47.

13. Bénézech $C$, et al. Lymphotoxin- $\beta$ receptor

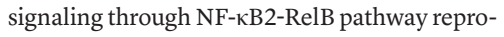
grams adipocyte precursors as lymph node stromal cells. Immunity. 2012;37(4):721-734.

14. del Rio ML, Schneider P, Fernandez-Renedo C, Perez-Simon JA, Rodriguez-Barbosa JI. LIGHT/ HVEM/LT $\beta$ R interaction as a target for the modulation of the allogeneic immune response in transplantation. Am J Transplant. 2013;13(3):541-551.

15. Estes JD. Pathobiology of HIV/SIV-associated changes in secondary lymphoid tissues. Immunol Rev. 2013;254(1):65-77.

16. Zeng M, Haase AT, Schacker TW. Lymphoid tissue structure and HIV-1 infection: life or death for T cells. Trends Immunol. 2012;33(6):306-314.
17. Maarouf $\mathrm{OH}$, et al. Repetitive ischemic injuries to the kidneys result in lymph node fibrosis and impaired healing. JCI Insight. 2018;3(13):120546.

18. Patel SD, Brennan G, Brazin J, Ciardiello AJ, Silver RB, Vannucci SJ. Mast cell isolation from the immature rat brain. Dev Neurosci. 2013;35(2-3):265-271.

19. He Z, et al. Degranulation of gastrointestinal mast cells contributes to hepatic ischemiareperfusion injury in mice. Clin Sci. 2018;132(20):2241-2259.

20. Jurewicz M, et al. Ischemic injury enhances dendritic cell immunogenicity via TLR4 and NF-kappa B activation. JImmunol. 2010;184(6):2939-2948.

21. Gan X, et al. Propofol attenuates small intestinal ischemia reperfusion injury through inhibiting NADPH oxidase mediated mast cell activation. Oxid Med Cell Longev. 2015;2015:167014.

22. Pejler G, Rönnberg E, Waern I, Wernersson S. Mast cell proteases: multifaceted regulators of inflammatory disease. Blood. 2010;115(24):4981-4990.

23. Jackson DG. Hyaluronan in the lymphatics: The key role of the hyaluronan receptor LYVE-1 in leucocyte trafficking. Matrix Biol. 2019;78-79:219-235.

24. Hinz B, Phan SH, Thannickal VJ, Galli A, Bochaton-Piallat ML, Gabbiani G. The myofibroblast: one function, multiple origins. Am J Pathol. 2007;170(6):1807-1816. 
25. Childs BG, Baker DJ, Wijshake T, Conover CA, Campisi J, van Deursen JM. Senescent intimal foam cells are deleterious at all stages of atherosclerosis. Science. 2016;354(6311):472-477.

26. Herro R, Da Silva Antunes R, Aguilera AR, Tamada K, Croft M. Tumor necrosis factor superfamily 14 (LIGHT) controls thymic stromal lymphopoietin to drive pulmonary fibrosis. J Allergy Clin Immunol. 2015;136(3):757-768.

27. Herro R, Croft M. The control of tissue fibrosis by the inflammatory molecule LIGHT (TNF Superfamily member 14). Pharmacol Res. 2016;104:151-155.

28. Tostanoski LH, et al. Reprogramming the local lymph node microenvironment promotes tolerance that is systemic and antigen specific. Cell Rep. 2016;16(11):2940-2952.

29. Katakai T, Kinashi T. Microenvironmental control of high-speed interstitial T cell migration in the lymph node. Front Immunol. 2016;7:194.

30. Jurewicz M, et al. Congenic mesenchymal stem cell therapy reverses hyperglycemia in experimental type 1 diabetes. Diabetes. 2010;59(12):3139-3147.

31. El Haddad N, et al. The novel role of SERPINB9 in cytotoxic protection of human mesenchymal stem cells. J Immunol. 2011;187(5):2252-2260.

32. Abdi R, Moore R, Sakai S, Donnelly CB, Mounayar M, Sackstein R. HCELL expression on murine MSC licenses pancreatotropism and confers durable reversal of autoimmune diabetes in NOD mice. Stem Cells. 2015;33(5):1523-1531.

33. Mounayar M, et al. PI3k $\alpha$ and STAT1 interplay regulates human mesenchymal stem cell immune polarization. Stem Cells. 2015;33(6):1892-1901.

34. Ben Nasr M, et al. Co-transplantation of autologous MSCs delays islet allograft rejection and generates a local immunoprivileged site. Acta Diabetol. 2015;52(5):917-927.

35. Bischoff SC. Role of mast cells in allergic and non-allergic immune responses: comparison of human and murine data. Nat Rev Immunol. 2007;7(2):93-104.

36. Galli SJ, Nakae S, Tsai M. Mast cells in the development of adaptive immune responses. Nat Immunol. 2005;6(2):135-142.

37. Theoharides TC, et al. Mast cells and inflammation. Biochim Biophys Acta. 2012;1822(1):21-33.

38. Bachelet I, Levi-Schaffer F. Mast cells as effector cells: a co-stimulating question. Trends Immunol. 2007;28(8):360-365.

39. Bachelet I, Levi-Schaffer F, Mekori YA. Mast cells: not only in allergy. Immunol Allergy Clin North Am. 2006;26(3):407-425.

40. Goes N, Urmson J, Ramassar V, Halloran PF. Ischemic acute tubular necrosis induces an extensive local cytokine response. Evidence for induction of interferon-gamma, transforming growth factor-beta 1, granulocyte-macrophage colony-stimulating factor, interleukin-2, and interleukin-10. Transplantation. 1995;59(4):565-572.

41. Halloran P, Aprile M. Factors influencing early renal function in cadaver kidney trans- plants. A case-control study. Transplantation 1988;45(1):122-127.

42. Szabo A, Heemann U. Ischemia reperfusion injury and chronic allograft rejection. Transplant Proc. 1998;30(8):4281-4284.

43. Kotsch K, et al. Improved long-term graft survival after HO-1 induction in brain-dead donors. Am J Transplant. 2006;6(3):477-486.

44. Land $\mathrm{W}$. The potential impact of the reperfusion injury on acute and chronic rejection events following organ transplantation. Transplant Proc. 1994;26(6):3169-3171.

45. Postalcioglu M, et al. Association of Cold Ischemia Time With Acute Renal Transplant Rejection. Transplantation. 2018;102(7):1188-1194.

46. Herrera J, Henke CA, Bitterman PB. Extracellular matrix as a driver of progressive fibrosis. JClin Invest. 2018;128(1):45-53.

47. Hinz B, Lagares D. Evasion of apoptosis by myofibroblasts: a hallmark of fibrotic diseases. Nat Rev Rheumatol. 2020;16(1):11-31.

48. Tomasek JJ, Gabbiani G, Hinz B, Chaponnier C, Brown RA. Myofibroblasts and mechano-regulation of connective tissue remodelling. Nat Rev Mol Cell Biol. 2002;3(5):349-363.

49. Klingberg F, et al. Prestress in the extracellular matrix sensitizes latent TGF- $\beta 1$ for activation. J Cell Biol. 2014;207(2):283-297.

50. Denton AE, Roberts EW, Linterman MA, Fearon DT. Fibroblastic reticular cells of the lymph node are required for retention of resting but not activated CD8 ${ }^{+}$T cells. Proc Natl Acad Sci USA. 2014;111(33):12139-12144.

51. Cremasco V, et al. B cell homeostasis and follicle confines are governed by fibroblastic reticular cells. Nat Immunol. 2014;15(10):973-981.

52. Estes JD, et al. Antifibrotic therapy in simian immunodeficiency virus infection preserves $\mathrm{CD}^{+}{ }^{+} \mathrm{T}$-cell populations and improves immune reconstitution with antiretroviral therapy. J Infect Dis. 2015;211(5):744-754.

53. Gregory JL, et al. Infection programs sustained lymphoid stromal cell responses and shapes lymph node remodeling upon secondary challenge. Cell Rep. 2017;18(2):406-418.

54. Fonseca DM, et al. Microbiota-dependent sequelae of acute infection compromise tissue-specific immunity. Cell. 2015;163(2):354-366.

55. Serrano M, Blasco MA. Putting the stress on senescence. Curr Opin Cell Biol. 2001;13(6):748-753.

56. Kuilman T, et al. Oncogene-induced senescence relayed by an interleukin-dependent inflammatory network. Cell. 2008;133(6):1019-1031.

57. Acosta JC, et al. Chemokine signaling via the CXCR2 receptor reinforces senescence. Cell. 2008;133(6):1006-1018.

58. Lasry A, Ben-Neriah Y. Senescence-associated inflammatory responses: aging and cancer perspectives. Trends Immunol. 2015;36(4):217-228.

59. van Deursen JM. The role of senescent cells in ageing. Nature. 2014;509(7501):439-446.

60. Tchkonia T, Zhu Y, van Deursen J, Campisi J, Kirkland JL. Cellular senescence and the senescent secretory phenotype: therapeutic opportuni- ties. J Clin Invest. 2013;123(3):966-972.

61. Acosta JC, et al. A complex secretory program orchestrated by the inflammasome controls paracrine senescence. Nat Cell Biol. 2013;15(8):978-990.

62. Chai Q, et al. Maturation of lymph node fibroblastic reticular cells from myofibroblastic precursors is critical for antiviral immunity. Immunity. 2013;38(5):1013-1024.

63. He S, Sharpless NE. Senescence in health and disease. Cell. 2017;169(6):1000-1011.

64. van de Pavert SA, Mebius RE. New insights into the development of lymphoid tissues. Nat Rev Immunol. 2010;10(9):664-674.

65. Piao W, et al. Regulation of T cell afferent lymphatic migration by targeting LT $\beta R$-mediated non-classical NFKB signaling. Nat Commun. 2018;9(1):3020.

66. Nakayama Y, Brinkman CC, Bromberg JS. Murine fibroblastic reticular cells from lymph node interact with $\mathrm{CD}^{+} \mathrm{T}$ cells through CD40-CD4OL. Transplantation. 2015;99(8):1561-1567.

67. Brinkman CC, et al. Treg engage lymphotoxin beta receptor for afferent lymphatic transendothelial migration. Nat Commun. 2016;7:12021.

68. Sibilano R, et al. A TNFRSF14-FceRI-mast cell pathway contributes to development of multiple features of asthma pathology in mice. Nat Commun. 2016;7:13696.

69. Shaikh RB, et al. Constitutive expression of LIGHT on T cells leads to lymphocyte activation, inflammation, and tissue destruction. JImmunol. 2001;167(11):6330-6337.

70. Uccelli A, Moretta L, Pistoia V. Mesenchymal stem cells in health and disease. Nat Rev Immunol. 2008;8(9):726-736.

71. Amé-Thomas P, et al. Human mesenchymal stem cells isolated from bone marrow and lymphoid organs support tumor B-cell growth: role of stromal cells in follicular lymphoma pathogenesis. Blood.2007;109(2):693-702.

72. Bromberg JS, et al. Gut microbiota-dependent modulation of innate immunity and lymph node remodeling affects cardiac allograft outcomes. JCI Insight. 2018;3(19):121045.

73. Knoblich K, et al. The human lymph node microenvironment unilaterally regulates T-cell activation and differentiation. PLoS Biol. 2018;16(9):e2005046.

74. Bahmani B, et al. Targeted delivery of immune therapeutics to lymph nodes prolongs cardiac allograft survival. J Clin Invest. 2018;128(11):4770-4786.

75. Corry RJ, Winn HJ, Russell PS. Heart transplantation in congenic strains of mice. Transplant Proc. 1973;5(1):733-735.

76. Overman EL, Rivier JE, Moeser AJ. CRF induces intestinal epithelial barrier injury via the release of mast cell proteases and TNF- $\alpha$. PLOS ONE. 2012;7(6):e39935.

77. Jurewicz M, et al. Donor antioxidant strategy prolongs cardiac allograft survival by attenuating tissue dendritic cell immunogenicity $\left({ }^{+}\right) . A m J$ Transplant. 2011;11(2):348-355. 